\title{
Estimation of the electron-phonon coupling parameter of Mo(110)-H and W(110)-H
}

\author{
J. Kröger \\ Institut für Experimentelle und Angewandte Physik, Christian-Albrechts-Universität zu Kiel, D-24098 Kiel, Germany
}

S. Lehwald and H. Ibach

Institut für Schichten und Grenzflächen, Forschungszentrum Jülich, D-52425 Jülich, Germany

(Received 18 February 2004; revised manuscript received 19 March 2004; published 24 May 2004)

\begin{abstract}
For the Kohn anomaly systems $\mathrm{Mo}(110)-(1 \times 1) \mathrm{H}$ and $\mathrm{W}(110)-(1 \times 1) \mathrm{H}$ we give an estimate of the electron-phonon coupling parameter. To this end we measured the energy of softened surface phonons at different temperatures by electron energy loss spectroscopy and exploited the fact that the Kohn anomaly weakens upon increasing the temperature. On the basis of the obtained coupling parameter we predict the critical temperature for the transition from the dynamically to the statically distorted lattice.
\end{abstract}

DOI: 10.1103/PhysRevB.69.201404

PACS number(s): 73.20.At, 63.20.Kr

The investigation of electron-phonon coupling is a central field of current solid state physics research. For instance, electron-phonon coupling in metals influences macroscopic properties like the specific heat or thermal and electric conductivity due to a renormalization of the electronic band dispersion close to the Fermi energy. ${ }^{1}$ Further, electron-phonon coupling is the underlying mechanism for conventional superconductivity: electrons are coupled to Cooper pairs by exchanging virtual phonons. ${ }^{2}$ The electron-phonon interaction has recently been shown to play an important role in the decay of metal electronic surface states. ${ }^{3}$ The strength of the interaction between electrons and phonons is given by the dimensionless electron-phonon coupling parameter $\lambda$, which originally was introduced to describe the band mass enhancement at the Fermi level. ${ }^{1}$

The most common procedure to extract the electronphonon coupling parameter experimentally is provided by the analysis of electron energy distribution curves by angleresolved photoelectron spectroscopy (ARPES). Under certain assumptions concerning the photoemission process the energy distribution of the photoelectrons recorded in the experiment corresponds to the spectral function of the photoholes. The spectral function contains the complex self-energy which describes the many-body physics involved in photoemission. The electron-phonon self-energy is one component. The experimental value of the electron-phonon coupling parameter is obtained either from the temperature dependence of the electron state linewidth (as given by the imaginary part of the electron-phonon self-energy) near the Fermi surface $^{4-8}$ or, equivalently, as the renormalization factor of the band dispersion at the Fermi level ${ }^{6,7,9,10}$ (governed by the real part of the electron-phonon self-energy). However, these procedures face the difficulty that they employ fairly simple phonon density of states, the Debye model for instance, which may not match the actual complexity of the system. Approaches to the coupling strength using other experimental techniques are scarce.

The main results of this article are (a) an estimation of the coupling constant for the Kohn anomaly systems $\mathrm{Mo}(110)-(1 \times 1) \mathrm{H}$ and $\mathrm{W}(110)-(1 \times 1) \mathrm{H}$ and $(\mathrm{b})$ the demonstration that electron energy loss spectroscopy (EELS) is another experimental method capable of extracting the electron-phonon coupling parameter. To our best knowledge the determination of the coupling constant involved in the Kohn anomaly of these systems is still lacking.

From helium atom scattering experiments ${ }^{11,12}$ and EELS investigations $^{13,14}$ it is known that these surfaces exhibit pronounced surface phonon anomalies: at several wave vectors the surface phonon dispersion curves reveal sharp indentations. Theory ${ }^{15}$ and ARPES experiments ${ }^{16,17}$ attribute these indentations to hydrogen-induced quasi-one-dimensional Fermi surface nesting. The spanning vectors which connect the nested Fermi surface contours (nesting vectors) equal the critical wave vectors of the affected surface phonons. Consequently, the observed softening of surface phonons could be unequivocally assigned to be the signature of a Kohn anomaly. Briefly speaking, the Kohn anomaly is induced by a screening singularity of the electron system for surface phonons with a critical wave vector $\mathbf{q}_{\mathrm{c}}=2 \mathbf{k}_{\mathrm{F}}$, where $\mathbf{k}_{\mathrm{F}}$ denotes the Fermi vector, i.e., the spanning vector between nested Fermi surface contours. The Kohn effect is enhanced if the system dimensionality is reduced and the Fermi surface resembles, locally in $k$ space, two parallel planes. The onedimensional situation is often referred to as perfect nesting which leads to the Peierls distortion, i.e., to the static rearrangement of lattice atoms. ${ }^{18}$ For this report we exploit another characteristic of the Kohn anomaly, namely its temperature dependence: upon lowering the temperature the softening of surface phonons at $\mathbf{q}_{\mathrm{c}}$ becomes more pronounced. For the one-dimensional free electron gas this behavior can be expressed analytically ${ }^{19}$ and will be used below. The electron-phonon coupling parameter we thus obtain describes the interaction between electronic surface states which belong to nested Fermi surface contours and acoustic surface phonons with a wave vector being equal to the nesting vector.

The experiment was performed under ultrahigh vacuum conditions (base pressure $5 \times 10^{-9} \mathrm{~Pa}$ ). Clean surfaces were obtained by repeated oxygen-annealing cycles at $1200 \mathrm{~K}$ and intermediate heating of the samples to $2200 \mathrm{~K}$. Cleanliness and crystalline order were monitored by dipole energy loss spectra and by low-energy electron diffraction (LEED). The custom-built spectrometer was operated with an energy resolution of $1.5 \mathrm{meV}$ and an angle resolution of better than $1^{\circ}$ 

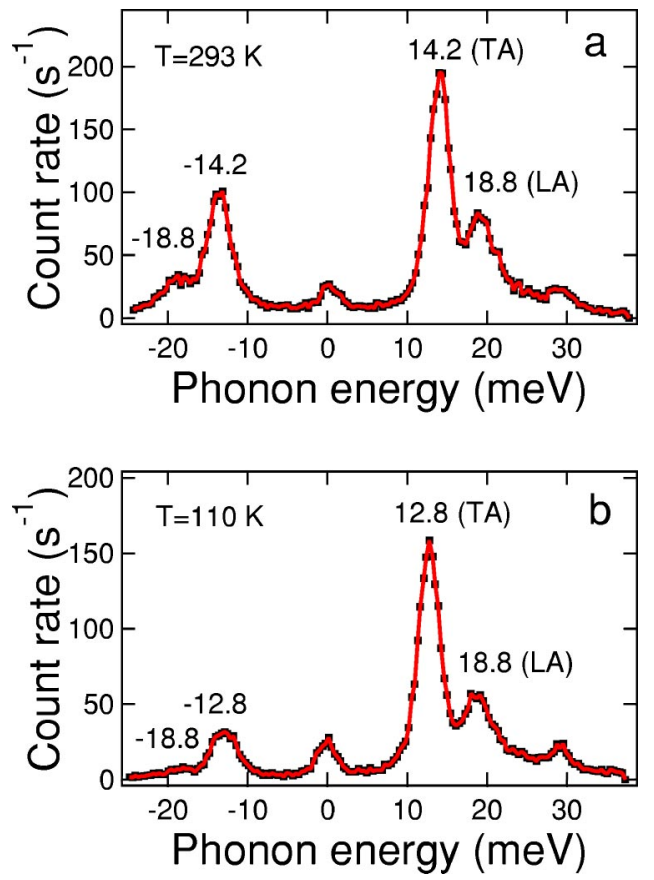

FIG. 1. (Color online) Substrate surface phonon spectra of $\mathrm{Mo}(110)-(1 \times 1) \mathrm{H}$ at (a) $293 \mathrm{~K}$ and at (b) $110 \mathrm{~K}$. The spectra were taken with an incident electron energy of $85 \mathrm{eV}$ and a momentum transfer of $0.9 \AA^{-1}$ along [001], i.e., the wave vector of the Kohn anomaly along this direction. Gain and loss sides of the spectra correspond to negative and positive energy values, respectively. Elastically scattered electrons give rise to the peak at zero energy. Then follow the transverse acoustic (TA) and the longitudinal acoustic (LA) surface phonon associated peaks. Upon decreasing the temperature the energy of the softened TA surface phonon is lowered, whereas an energy lowering of the LA surface phonon stays below the detection limit of the spectrometer. The full line results from smoothing the raw data (black dots).

(corresponding to a wave vector resolution of better than $0.04 \AA^{-1}$ for the applied impact electron energies). Using liquid nitrogen the sample surfaces can be cooled down to $110 \mathrm{~K}$. Adsorption of atomic hydrogen was performed by exposing the liquid-nitrogen cooled clean surfaces to $\mathrm{H}_{2}$ gas of $99.995 \%$ purity. The $(1 \times 1)$ saturation superstructure is obtained at 1 monolayer (ML) coverage and is monitored by sharp diffraction spots in the LEED pattern, and sharp and intense loss peaks in specular EELS spectra, which do not alter upon further increasing the exposure.

For extracting the electron-phonon coupling parameter which leads to the Kohn anomaly the energy of the softened surface phonons must be measured for at least two different temperatures (see the discussion below). Phonon spectra at different temperatures are presented in Figs. 1(a) and 1(b). For both spectra the energy of incident electrons was set to $85 \mathrm{eV}$ and the analyzer of the instrument was rotated out of specular reflection to detect electrons which transferred a momentum of $q_{\mathrm{c}}=0.9 \AA^{-1}$, which is the critical wave vector of the Kohn anomaly along [001]. ${ }^{14}$ The sample temperatures are $293 \mathrm{~K}$ [Fig. 1(a)] and $110 \mathrm{~K}$ [Fig. 1(b)]. The peak at zero energy is associated to elastically scattered electrons, whereas the peaks on the positive (negative) energy axes correspond to energy losses (gains) of the scattered electrons due to surface phonons. In particular, the transverse acoustic (TA) and longitudinal acoustic (LA) surface phonons give rise to loss peaks at 14.2 and $18.8 \mathrm{meV}$ in Fig. 1(a), respectively, and at 12.8 and $18.8 \mathrm{meV}$ in Fig. 1(b), respectively. By comparing Figs. 1(a) and 1(b) it is obvious that the energy of the softened TA surface phonon is lowered from 14.2 $\mathrm{meV}$ at $293 \mathrm{~K}$ down to $12.8 \mathrm{~K}$ at $110 \mathrm{~K}$. We attribute this energy shift to the temperature dependence of the Kohn anomaly. In particular, we rule out any temperature-induced change of the adsorbate structure which was controlled by LEED and dipole spectra using EELS. For the softened LA surface phonon a shift of the energy upon decreasing the temperature stayed below the detection limit of the spectrometer. From ARPES measurements we know that hydrogen-induced quasi-one-dimensional nesting of Fermi surface contours of surface states is present for $\mathrm{Mo}(110)-(1 \times 1) \mathrm{H}^{17}$ and $\mathrm{W}(110)-(1 \times 1) \mathrm{H}^{16}$ The nested contours involved in the softening of the surface phonons extend in $k$ space for $\approx 0.6 \AA^{-1}$ along $[1 \overline{1} 0]^{16,17}$ which corresponds to $\approx 21 \%$ of the extension of the surface Brillouin zone along this direction and demonstrates clearly the local one-dimensional situation. Also less pronounced nesting was demonstrated to lead to one-dimensional behavior, e.g., the proposed charge density wave (CDW) transition in $\mathrm{Bi}(111) .{ }^{20}$ Further, the electronic surface state bands reveal a parabolic dispersion curve along [001] indicating a free-electron like behavior. ${ }^{16,17}$ Thus, in order to extract the electron-phonon coupling parameter, we were motivated to apply an analytic expression derived for the one-dimensional free electron gas which gives the temperature dependence of the softened phonon energy $E(T),{ }^{19}$

$$
E^{2}(T)=E_{0}^{2}\left[1-\lambda \ln \left(\frac{1.14 E_{\mathrm{F}}}{k_{\mathrm{B}} T}\right)\right] .
$$

In Eq. (1) $E_{0}$ denotes the phonon energy of the bare phonon at the critical wave vector, i.e., the energy which is not renormalized due to electron-phonon coupling; $E_{\mathrm{F}}$ is the Fermi energy, and $k_{\mathrm{B}}$ is Boltzmann's constant. In order to be independent of $E_{0}$ (since we do not have access to the bare phonon energy) we write down Eq. (1) for two different temperatures $T_{1}<T_{2}$ and arrange the equations to obtain

$$
\lambda=\frac{E^{2}\left(T_{2}\right)-E^{2}\left(T_{1}\right)}{E^{2}\left(T_{2}\right) \ln \left(\frac{1.14 E_{\mathrm{F}}}{k_{\mathrm{B}} T_{1}}\right)-E^{2}\left(T_{1}\right) \ln \left(\frac{1.14 E_{\mathrm{F}}}{k_{\mathrm{B}} T_{2}}\right)} .
$$

With $E\left(T_{1}=110 \mathrm{~K}\right)=12.8 \mathrm{meV}, \quad E\left(T_{2}=293 \mathrm{~K}\right)$ $=14.2 \mathrm{meV}$, and $E_{\mathrm{F}}=860 \mathrm{meV}$ (which is the bottom edge of the electron band taking part in the quasi-one-dimensional Fermi surface nesting $)^{17}$ we obtain $\lambda=0.11 \pm 0.02$ for $\mathrm{Mo}(110)-(1 \times 1) \mathrm{H}$ [considering the experimental accuracy of measured quantities entering Eq. (2), i.e., $0.2 \mathrm{meV}^{21}$ for the phonon energies $E(T), 3 \mathrm{~K}$ for the temperature $T$, and 30 $\mathrm{meV}$ for $\left.E_{\mathrm{F}}\right]$. With decreasing temperature the renormalized phonon energy goes to zero, which defines a transition temperature $T_{\mathrm{c}}$ when a static lattice distortion occurs. From Eq. (1) with $E\left(T_{\mathrm{c}}\right)=0$ we find ${ }^{19}$ 
TABLE I. Extracted electron-phonon coupling parameters $\lambda$ for the Kohn anomaly systems $\mathrm{Mo}(110)-(1 \times 1) \mathrm{H}$ and $\mathrm{W}(110)-(1$ $\times 1)$ H. $E_{\mathrm{c}}(T)$ gives the energy of the softened transverse acoustic surface phonon at the specified temperature.

\begin{tabular}{lccc}
\hline \hline & $E_{\mathrm{c}}(293 \mathrm{~K})(\mathrm{meV})$ & $E_{\mathrm{c}}(110 \mathrm{~K})(\mathrm{meV})$ & $\lambda$ \\
\hline $\mathrm{Mo}(110)-\mathrm{H}$ & $14.2 \pm 0.2$ & $12.8 \pm 0.2$ & $0.11 \pm 0.02$ \\
$\mathrm{~W}(110)-\mathrm{H}$ & $10.6 \pm 0.2$ & $9.2 \pm 0.2$ & $0.13 \pm 0.02$ \\
\hline \hline
\end{tabular}

$$
k_{\mathrm{B}} T_{\mathrm{c}}=1.14 E_{\mathrm{F}} \exp \left(-\frac{1}{\lambda}\right) .
$$

With the values at hand and the calculated errors we estimate within the applied model that the (dynamic) Kohn anomaly for $\mathrm{Mo}(110)-(1 \times 1) \mathrm{H}$ leads to a static lattice distortion between 0 and $4 \mathrm{~K}$. For $\mathrm{W}(110)-(1 \times 1) \mathrm{H}$ we find $E\left(T_{1}\right.$ $=110 \mathrm{~K})=9.2 \mathrm{meV}^{13} E\left(T_{2}=293 \mathrm{~K}\right)=10.6 \mathrm{meV}^{13}$ and $E_{\mathrm{F}}=(1300 \pm 100) \mathrm{eV}$ (bottom band edge of electrons belonging to the nested Fermi surface contours). ${ }^{22}$ The resulting electron-phonon coupling parameter is $\lambda=0.13 \pm 0.02$ and the static lattice distortion is predicted to occur between 0 and $16 \mathrm{~K}$. We summarized the values for Mo(110)-(1 $\times 1) \mathrm{H}$ and $\mathrm{W}(110)-(1 \times 1) \mathrm{H}$ in Table I. Fermi-surface averaged values of the electron-phonon coupling parameter for bulk Mo are found to be $\approx 0.4 .^{1,23}$ For the clean $\operatorname{Mo}(110)$ surface state Valla et al. ${ }^{7}$ found $\lambda=0.35$ and $\lambda=0.52$ for two different binding energies. We are not aware of calculated or experimentally measured coupling constants for Mo(110)-H. The Fermi-surface averaged electron-phonon coupling parameter for bulk $\mathrm{W}$ was calculated to give $\approx 0.19 .^{24}$ In a recent ARPES study of $\mathrm{W}(110)-(1 \times 1) \mathrm{H}$ the coupling between electronic surface states and hydrogen adsorbate phonons was examined. ${ }^{25}$ The authors demonstrated that the electron-phonon coupling parameter is $\approx 0.1$ and $\approx 0.8$ for spin-orbit split electronic surface states. In a further analysis of this adsorbate system the authors showed that depending on the position on the Fermi surface contours of the spinorbit split surface states the electron-phonon coupling strength varies from zero to the maximum values given above. ${ }^{26}$ From our vibrational spectroscopy data we extracted coupling parameters of $\approx 0.11$ and $\approx 0.13$ for $\mathrm{Mo}(110)-\mathrm{H}$ and $\mathrm{W}(110)-\mathrm{H}$, respectively. The experimental fact that our values are lower than the coupling parameters characterizing the interaction strength between electrons and adsorbate phonons ${ }^{25,26}$ can be attributed to the degree of surface localization of the vibrational excitations. For the interaction between surface state electrons and surface phonons the spatial overlap between the surface state wave function and the phonon eigenvector plays an important role. For acoustic substrate surface phonons it is a well-established fact that penetration of the vibration amplitude into the bulk increases with decreasing wave vectors parallel to the surface. The Kohn anomaly along [001] occurs at wave vectors well apart from the surface Brillouin zone boundary (located at $\approx 1.5 \AA^{-1}$ along [001]). Furthermore, the electron-phonon coupling parameter involving zero momentum adsorbate phonons is not necessarily related to the one involving finite momentum acoustic substrate surface phonons. Finally, the Kohn anomaly represents a dynamic softening of surface phonons, i.e., the electron-phonon coupling is expected to be lower than the one encountered for the static lattice distortion. $^{27}$ The latter is usually accompanied by a CDW formation. Recently, experimental evidence for a Peierls-type CDW was reported for $\mathrm{Bi}(111)$ by $\mathrm{ARPES}^{20}$ The corresponding electron-phonon coupling parameter was determined to be $\approx 0.6{ }^{10}$ The family of layered $2 \mathrm{H}$ transition metal dichalcogenides represent CDW systems, where for $2 \mathrm{H}-\mathrm{NbSe}_{2}$ electron-phonon coupling parameters between $0.3^{28}$ and $0.6^{29}$ were reported. Further ARPES studies which demonstrate that the electron-phonon coupling parameter depends strongly on the wave vector and the binding energy are reported in Refs. 30 and 31. For instance, the electronphonon coupling parameter for $\mathrm{Bi}(100)$ ranges from 0.1 to 0.7 in a binding energy interval between 0.1 and $0.3 \mathrm{eV}^{31}$ Even lower electron-phonon coupling constants, ranging from $\approx 0.02$ to $\approx 0.08$, have been obtained for transitionmetal diborides $\mathrm{TB}_{2}(\mathrm{~T}=\mathrm{Zr}, \mathrm{Nb}, \mathrm{Ta})$ by point contact spectroscopy. ${ }^{32}$ On the basis of our experimental data we find that the coupling parameter between electrons and the LA surface phonon must be lower than the one found for the TA surface phonon. At present an explanation for this observation is not available.

In conclusion, we extracted the electron-phonon coupling parameter for the Kohn anomaly systems Mo(110)-(1 $\times 1) \mathrm{H}$ and $\mathrm{W}(110)-(1 \times 1) \mathrm{H}$ by analyzing temperaturedependent surface phonon spectra at the critical wave vector using electron energy loss spectroscopy. The obtained coupling parameter gives the interaction strength between substrate surface phonons affected by the Kohn anomaly and electrons which belong to nested Fermi surface contours. We thus demonstrated that employing EELS for temperaturedependent surface phonon spectroscopy of Kohn anomaly systems is an alternative way to determine the electronphonon coupling parameter. On the basis of the obtained coupling constants we estimated the transition temperature at which the dynamic lattice distortion turns into a static one.
${ }^{1} \mathrm{G}$. Grimvall, The Electron-Phonon Interaction in Metals (NorthHolland, Amsterdam, 1981).

${ }^{2}$ J. Bardeen, L.N. Cooper, and J.R. Schrieffer, Phys. Rev. 108, 1175 (1957).

${ }^{3}$ A. Eiguren, B. Hellsing, F. Reinert, G. Nicolay, E.V. Chulkov,

V.M. Silkin, S. Hüfner, and P.M. Echenique, Phys. Rev. Lett. 88,
066805 (2002)

${ }^{4}$ B.A. McDougall, T. Balasubramanian, and E. Jensen, Phys. Rev. B 51, 13891 (1995).

${ }^{5} \mathrm{Ph}$. Hofmann, Y.Q. Cai, Ch. Grütter, and J.H. Bilgram, Phys. Rev. Lett. 81, 1670 (1998).

${ }^{6}$ M. Hengsberger, D. Purdie, P. Segovia, M. Garnier, and Y. Baer, 
Phys. Rev. Lett. 83, 592 (1999).

${ }^{7}$ T. Valla, A.V. Fedorov, P.D. Johnson, and S.L. Hulbert, Phys. Rev. Lett. 83, 2085 (1999).

${ }^{8}$ F. Reinert, B. Eltner, G. Nicolay, D. Ehm, S. Schmidt, and S. Hüfner, Phys. Rev. Lett. 91, 186406 (2003).

${ }^{9}$ S. LaShell, E. Jensen, and T. Balasubramanian, Phys. Rev. B 61, 2371 (2000).

${ }^{10}$ C.R. Ast and H. Höchst, Phys. Rev. B 66, 125103 (2002).

${ }^{11}$ E. Hulpke and J. Lüdecke, Phys. Rev. Lett. 68, 2846 (1992).

${ }^{12}$ E. Hulpke and J. Lüdecke, Surf. Sci. 287/288, 837 (1993).

${ }^{13}$ M. Balden, S. Lehwald, and H. Ibach, Phys. Rev. B 53, 7479 (1996).

${ }^{14}$ J. Kröger, S. Lehwald, and H. Ibach, Phys. Rev. B 55, 10895 (1997).

${ }^{15}$ B. Kohler, P. Ruggerone, S. Wilke, and M. Scheffler, Phys. Rev. Lett. 74, 1387 (1995).

${ }^{16}$ E. Rotenberg and S.D. Kevan, Phys. Rev. Lett. 80, 2905 (1998).

${ }^{17}$ J. Kröger, T. Greber, and J. Osterwalder, Phys. Rev. B 61, 14146 (2000).

${ }^{18}$ R.E. Peierls, Quantum Theory of Solids (Clarendon, Oxford, 1955).

${ }^{19}$ G. Grüner, Density Waves in Solids (Perseus, Cambridge, MA, 1994).

${ }^{20}$ C.R. Ast and H. Höchst, Phys. Rev. Lett. 90, 016403 (2003).

${ }^{21}$ The accuracy of the energy position results from averaging more than 100 spectra using impact energies in the range of $20-200$ $\mathrm{eV}$.

${ }^{22}$ M. Hochstrasser, J.G. Tobin, E. Rotenberg, and S.D. Kevan, Phys. Rev. Lett. 89, 216802 (2002).

${ }^{23}$ S.Y. Savrasov and D.Y. Savrasov, Phys. Rev. B 54, 16487 (1996).

${ }^{24}$ W. John, V.V. Nemoshkalenko, V.N. Antonov, and V.N. Antonov, Phys. Status Solidi B 121, 233 (1984).

${ }^{25}$ E. Rotenberg, J. Schaefer, and S.D. Kevan, Phys. Rev. Lett. 84, 2925 (2000).

${ }^{26}$ E. Rotenberg and S.D. Kevan, J. Electron Spectrosc. Relat. Phenom. 126, 125 (2002).

${ }^{27}$ E. Tosatti, in Electronic Surface and Interface States on Metallic Systems, edited by E. Bertel and M. Donath (World Scientific, Singapore, 1995).

${ }^{28}$ R. Corcoran, P. Meeson, Y. Onuki, P.-A. Probst, M. Springford, K. Takita, H. Harima, G.Y. Guo, and B.L. Gyorffy, J. Phys.: Condens. Matter 6, 4479 (1994).

${ }^{29}$ T. Valla, A.V. Fedorov, P.D. Johnson, P.-A. Glans, C. McGuinness, K.E. Smith, E.Y. Andrei, and H. Berger, Phys. Rev. Lett. 92, 086401 (2004).

${ }^{30}$ D.-A. Luh, T. Miller, J.J. Paggel, and T.-C. Chiang, Phys. Rev. Lett. 88, 256802 (2002).

${ }^{31}$ J.E. Gayone, S.V. Hoffmann, Z. Li, and Ph. Hofmann, Phys. Rev. Lett. 91, 127601 (2003).

${ }^{32}$ Y.G. Naidyuk, O.E. Kvitnitskaya, I.K. Yanson, S.-L. Drechsler, G. Behr, and S. Otani, Phys. Rev. B 66, 140301 (2002). 